\title{
A nearly real-time high temperature laser-plasma diagnostic using photonuclear reactions in tantalum
}

I. Spencer ${ }^{\mathrm{a})}$

Department of Physics and Astronomy, University of Glasgow, Glasgow G12 8QQ, United Kingdom

K. W. D. Ledingham

Department of Physics and Astronomy, University of Glasgow, Glasgow G12 8QQ and AWE plc, Aldermaston, Reading RG7 4PR, United Kingdom

R. P. Singhal, T. McCanny, and P. McKenna

Department of Physics and Astronomy, University of Glasgow, Glasgow G12 8QQ, United Kingdom

E. L. Clark

Blackett Laboratory, Imperial College, Prince Consort Road, London SW7 2BZ and AWE plc, Aldermaston, Reading RG7 4PR, United Kingdom

K. Krushelnick, M. Zepf, F. N. Beg, M. Tatarakis, and A. E. Dangor

Blackett Laboratory, Imperial College, Prince Consort Road, London SW7 2BZ, United Kingdom

R. D. Edwards and M. A. Sinclair

AWE plc, Aldermaston, Reading RG7 4PR, United Kingdom

P. A. Norreys, R. J. Clarke, and R. M. Allott

Rutherford Appleton Laboratory, Chilton, Oxon., OX11, OQX, United Kingdom

(Received 28 May 2002; accepted 5 August 2002)

A method of measuring the temperature of the fast electrons produced in ultraintense laser-plasma interactions is described by inducing photonuclear reactions, in particular $(\gamma, n)$ and $(\gamma, 3 n)$ reactions in tantalum. Analysis of the $\gamma$ rays emitted by the daughter nuclei of these reactions using a germanium counter enables a relatively straightforward near real-time temperature measurement to be made. This is especially important for high temperature plasmas where alternative diagnostic techniques are usually difficult and time consuming. This technique can be used while other experiments are being conducted. (C) 2002 American Institute of Physics.

[DOI: $10.1063 / 1.1511802]$

\section{INTRODUCTION}

Throughout the last $3 \mathrm{yr}$, ultraintense lasers have been used to generate photonuclear reactions. ${ }^{1-5}$ This has been made possible since electrons can be accelerated to energies as high as $100 \mathrm{MeV}$ when a laser of intensity $10^{20} \mathrm{~W} \mathrm{~cm}^{-2}$ interacts with matter. These relativistic electrons can be converted to $\gamma$ rays via bremsstrahlung in a thick, high $Z$ radiator, and these high-energy photons are then capable of producing photonuclear reactions, typically $(\gamma, m n)(m$ $=1,2,3, \ldots)$ and $(\gamma$, fission $)$ reactions. The experimental verification of laser induced nuclear fission ${ }^{3-5}$ was based on a concept proposed by Boyer $\mathrm{et} \mathrm{al.}^{6}$ in 1988. Possible applications of this work are using a table-top laser as a fission ignition device, ${ }^{3}$ and performing "table-top nuclear physics" experiments. In addition, it has been shown that energetic ion beams, in particular proton beams, are generated in ultraintense laser-matter interactions. ${ }^{7-10}$ In the most recent experiments, ${ }^{11-19}$ multi-MeV protons have been observed for laser irradiances up to and exceeding $I \lambda^{2}=10^{20}$ $\mathrm{W} \mathrm{cm}{ }^{-2} \mu \mathrm{m}^{2}$, where $I$ is the laser intensity in $\mathrm{W} \mathrm{cm}^{-2}$ and $\lambda$

\footnotetext{
a) Author to whom correspondence should be addressed; electronic mail: i.spencer@physics.gla.ac.uk
}

is the laser wavelength in $\mu \mathrm{m}$. These proton beams have been used to induce nuclear reactions in materials, typically $(p, n)$ and $(p, \alpha)$ reactions in order to produce short-lived positron emitting radioisotopes that could potentially be used in nuclear medicine, specifically positron emission tomography. ${ }^{14,16}$ A possible extension of this work would be to produce commercial quantities of positron emitting radioisotopes using a table-top laser. Nemoto et al. ${ }^{18}$ have recently demonstrated table-top laser production of the isotope ${ }^{11} \mathrm{C}$, using laser-accelerated deuteron beams.

Another important application of this new technology is the use of nuclear reactions to diagnose fundamental parameters of the laser-plasma interaction. For example, the direction of fast electrons produced in the interaction has been measured under various laser conditions, ${ }^{20}$ and also $(p, n)$ reactions on copper stacks has enabled measurement of the flux and energies of the protons produced in the interaction. ${ }^{11,16,19}$ In addition, $(\gamma, n)$ reactions have been used to measure the temperature of the fast electrons produced in the interaction at a preliminary stage ${ }^{4}$ using copper and carbon samples. This was made possible because the reaction thresholds or $Q$ values of $(\gamma, n)$ reactions in copper and carbon are different. In other studies, ${ }^{2,21}$ photonuclear reactions in gold were used to provide information about the 
$\mathrm{MeV}$ photon flux and energies produced in petawatt lasersolid interactions.

This article describes a method of measuring the temperature of the fast electrons produced in ultraintense laserplasma interactions using a single tantalum target, by utilizing $(\gamma, n)$ and $(\gamma, 3 n)$ reactions in tantalum and measuring the radiation emitted by the products of these reactions via $\gamma$-ray spectroscopy. By measuring the relative activities of the products of reactions with different $Q$ values, temperature measurements can made by calculating a theoretical value for these activity ratios for a given electron temperature. The technique should yield results on large laser systems on a shot-to-shot basis, and it is possible to carry out this process while other experiments are being conducted, since all one requires is a tantalum sample placed in the direction of the fast electron beam. This means that the technique can be employed whether the target is solid or gaseous, as long as the energies of the electrons produced are sufficiently high to induce the $(\gamma, 3 n)$ reaction, as will be described below. That is, the technique is applicable for laser irradiances exceeding the value $I \lambda^{2}=10^{19} \mathrm{~W} \mathrm{~cm}^{-2} \mu \mathrm{m}^{2}$ where alternative plasma diagnostics are difficult. This technique has been applied at a preliminary level ${ }^{22}$ in the characterization of a self-modulated laser wakefield accelerator, i.e., in underdense laser-plasma interactions. The technique is described in greater detail herein.

\section{EXPERIMENT}

The experiment employed the ultraintense beam line of the VULCAN Nd:glass laser system $^{23}$ at the Rutherford Appleton Laboratory, UK, operating in the chirped pulse amplification $^{24,25}$ mode. The beam was incident on a tantalum target, of dimensions $10 \times 10 \mathrm{~mm}$ and $1.75 \mathrm{~mm}$ thick, and at an angle of $45^{\circ}$. The light was $p$ polarized, and the target was maintained in a vacuum of $\sim 10^{-4}$ Torr. When operated in this manner, the laser delivered pulses of energy up to $120 \mathrm{~J}$ and 1 ps duration. A 95\% reflecting turning mirror located after the compression gratings was used to steer the beam on target, focused by a $F 4,450 \mathrm{~mm}$ focal length off-axis parabolic mirror. The turning mirror permitted 5\% transmission of the laser energy to diagnose the laser pulse. The pulse duration was measured by a single shot autocorrelator, and the focal spot quality by an equivalent plane monitor. Equivalent plane images show that the focal spot was $6 \mu \mathrm{m}$, which yields intensities of up to $10^{20} \mathrm{~W} \mathrm{~cm}^{-2}$. A more detailed description of the experimental setup and the principal diagnostics involved can be found in Ref. 1. The main-pulse/prepulse contrast ratio was measured by a third-order autocorrelator and found to be $1: 10^{-6}$. The prepulse intensity is sufficient to ionize the target surface and generate a plasma on the target surface which expands outward prior to the arrival of the main pulse. The main pulse then interacts with this preplasma, and accelerates free electrons into the target. These relativistic electrons with an energy distribution proportional to $E^{2} \exp (-E / k T)^{1,4,26}$ (where $E$ is the electron energy, $k$ is the Boltzmann constant, and $T$ is the electron temperature) are then slowed down in the high $Z$ tantalum target, and generate
TABLE I. Nuclear reaction data for the reactions studied.

\begin{tabular}{|c|c|c|c|c|}
\hline Reaction & $\begin{array}{l}\text { Half life } \\
\text { (h) }\end{array}$ & $-Q(\mathrm{MeV})$ & Peak $\sigma(\mathrm{mb})$ & $\begin{array}{c}\text { Principal } \\
\text { radiations }\end{array}$ \\
\hline${ }^{181} \mathrm{Ta}(\gamma, n){ }^{180} \mathrm{Ta}$ & 8.1 & 7.6 & 360 & $\begin{array}{c}\text { Hf } \mathrm{x} \text { rays } \\
\gamma \text { rays } \\
93 \mathrm{keV}(4 \%) \\
103 \mathrm{keV}(0.78 \%)\end{array}$ \\
\hline${ }^{181} \mathrm{Ta}(\gamma, 3 n){ }^{178 m} \mathrm{Ta}$ & 2.36 & 22.1 & 20 & $\begin{array}{c}\text { Hf x rays } \\
\gamma \text { rays } \\
89 \mathrm{keV}(54 \%) \\
93 \mathrm{keV}(14 \%) \\
214 \mathrm{keV}(75 \%) \\
328 \mathrm{keV}(120 \% \\
\text { complex) } \\
426.5 \mathrm{keV}(97 \%)\end{array}$ \\
\hline
\end{tabular}

$\gamma$-ray photons via bremsstrahlung. The bremsstrahlung photons then interact with the tantalum nuclei, and induce photonuclear reactions. Once the tantalum was irradiated with the VULCAN pulse, the target chamber was restored to atmospheric pressure and the tantalum target was extracted. The time taken to restore the chamber to atmospheric pressure was a few minutes, after which the sample was transferred to a nuclear laboratory for analysis. This limited the measurable lifetimes of the unstable isotopes produced to a few minutes or more. The $\gamma$ radiation emitted from the tantalum target was measured in an intrinsic germanium detector. The efficiency of the detector was calibrated as a function of energy using sources of known energy and activity $\left({ }^{57} \mathrm{Co},{ }^{22} \mathrm{Na},{ }^{137} \mathrm{Cs},{ }^{60} \mathrm{Co}\right.$, and $\left.{ }^{152} \mathrm{Eu}\right)$. This made it possible to measure the energy and activity of any $\gamma$ rays incident on the detector.

\section{RESULTS}

The tantalum target was composed of $100 \%{ }^{181} \mathrm{Ta}$, the stable tantalum isotope. The two reactions of interest are $\left.{ }^{181} \mathrm{Ta}(\gamma, n)\right)^{180} \mathrm{Ta}$ and ${ }^{181} \mathrm{Ta}(\gamma, 3 n){ }^{178 m} \mathrm{Ta} .{ }^{180} \mathrm{Ta}$ decays via electron capture with a half life of $8.1 \mathrm{~h}$ to an excited state of ${ }^{180} \mathrm{Hf}$, which then achieves stability via the emission of a $\gamma$ ray of $93 \mathrm{keV}$. Hf $\mathrm{x}$ rays are also emitted in the decay $\left(K_{\alpha}\right.$ $=55.7 \mathrm{keV}, K_{\beta}=63.2 \mathrm{keV}$ ). The competing decay to EC is $\beta^{-}$, again with a characteristic half life of $8.1 \mathrm{~h}$. This produces an excited state of ${ }^{180} \mathrm{~W}$, which achieves stability via the emission of a $\gamma$ ray of $103 \mathrm{keV}$ plus $\mathrm{Hf} \mathrm{x}$ rays. ${ }^{27,28}$

${ }^{178 m} \mathrm{Ta}$ decays via EC with a characteristic half life of $2.36 \mathrm{~h}$ to excited states of ${ }^{178} \mathrm{Hf}$, which then achieves stability via the emission of a number of $\gamma$ rays of various energies plus Hf x rays. The $Q$ values, half lives, peak cross sections, and principal radiations of these reactions are summarized in Table I, and the relevant portions of the decay schemes for ${ }^{180} \mathrm{Ta}$ and ${ }^{178 m} \mathrm{Ta}$ are shown in Fig. 1. The cross sections for these reactions are shown in Fig. $2 .{ }^{29}$

The product of the reaction ${ }^{181} \mathrm{Ta}(\gamma, 2 n){ }^{179} \mathrm{Ta}(Q=$ $-14.2 \mathrm{MeV}$ ) has a half life of $600 \mathrm{~d}$, which is too long for the type of analysis undertaken in this study, and the principal radiation is $\mathrm{Hf} \mathrm{x}$ rays, making it impossible to distinguish from other $(\gamma, m n)$ reactions. This was why the reaction was 

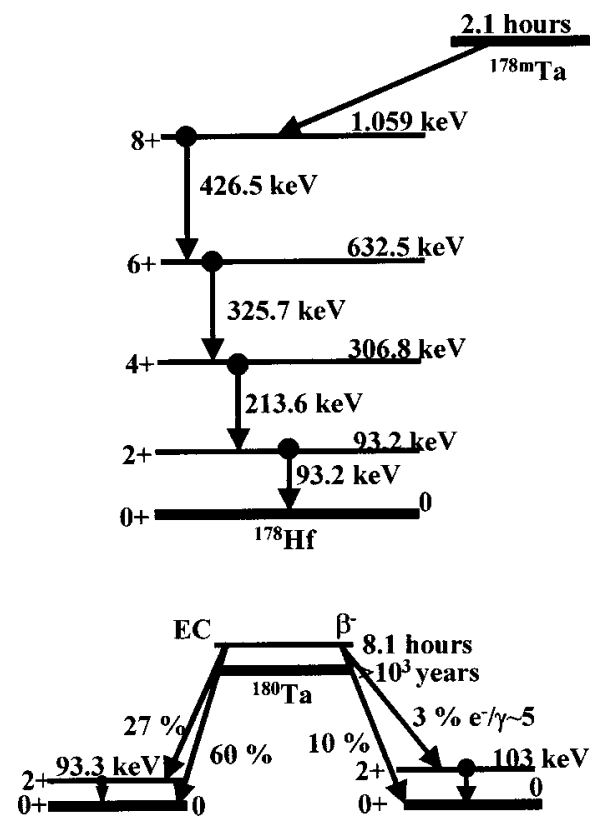

FIG. 1. Relevant decay schemes for ${ }^{180} \mathrm{Ta}$ and ${ }^{178 m} \mathrm{Ta}$.

not studied in detail in the present case, although the cross section for this reaction is shown in Fig. 2 for comparison with the $(\gamma, n)$ and $(\gamma, 3 n)$ processes.

The tantalum sample was analyzed using the germanium detector described above which was shielded in lead and coupled to a multichannel analyzer. The sample was measured for $1 \mathrm{~h}$. It is also important to measure the contribution from any background radiation. The background spectrum was taken over $12 \mathrm{~h}$ to improve the counting statistics, then the overall counting rate was divided by 12 to show its strength relative to the tantalum signal. Figure 3 shows a typical spectrum obtained in the region $0-500 \mathrm{keV}$ with the background contribution overlapped. Many lines are observed, most notably the $93.6 \mathrm{keV}$ peak (accepted value 93.3 $\mathrm{keV}$ ) which is emitted by both ${ }^{180} \mathrm{Ta}$ and ${ }^{178 m} \mathrm{Ta}$, and the $103.3 \mathrm{keV}$ peak which is emitted solely by ${ }^{180} \mathrm{Ta}$.

A strong case for the presence of ${ }^{178 m}$ Ta can be made by examining an interesting feature of the decay scheme for this isotope. $^{27,28}$ There is a cascade of transitions from the $8+$ level of ${ }^{178} \mathrm{Hf}$ to $6+, 4+, 2+$, then to the ground state via the emission of $\gamma$ rays of energies 426.5, 325.7, 213.6, and 93.2 $\mathrm{keV}$. The intensities of these peaks are all equal, and these peaks are evident in Fig. 3 (experimentally measured values

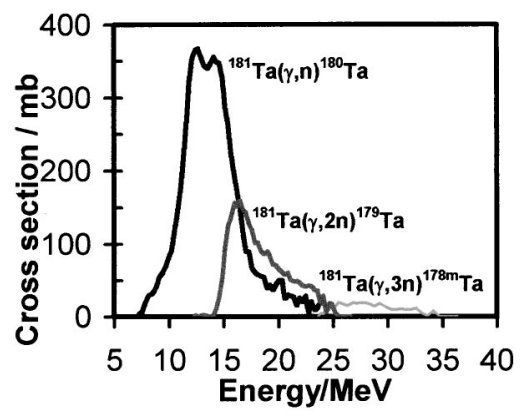

FIG. 2. Experimentally measured cross sections (Ref. 29) for the reactions studied.

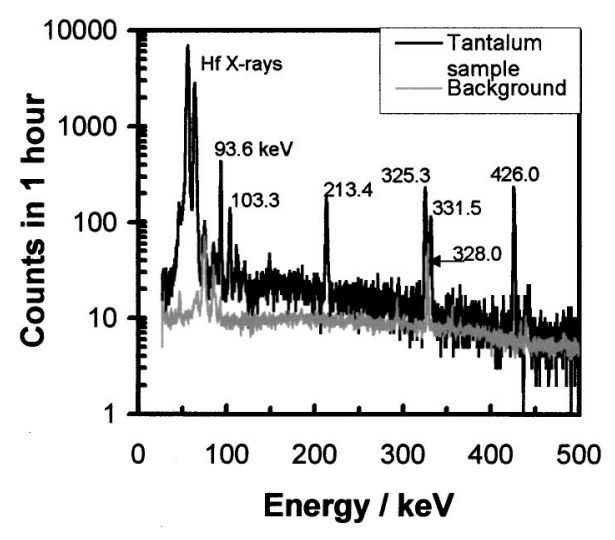

FIG. 3. Tantalum spectrum in the region $0-500 \mathrm{keV}$. Many $\gamma$-ray lines are evident.

426.0, 325.3, 213.4, and $93.6 \mathrm{keV}$ ). This gives an automatic efficiency calibration for the detector, in addition to the measurement made using calibration sources. These lines are also a fingerprint and evidence of the presence of ${ }^{178 m} \mathrm{Ta}$. Even though the $93.6 \mathrm{keV}$ peak has a contribution from ${ }^{180} \mathrm{Ta}$, the observation of the other three lines provides proof of the presence of ${ }^{178 m} \mathrm{Ta}$, meaning that $\gamma$ rays of energy greater than $22 \mathrm{MeV}$ [the energy threshold for the reaction $\left.{ }^{181} \mathrm{Ta}(\gamma, 3 n){ }^{178 m} \mathrm{Ta}\right]$ were produced in the laser-plasma interaction.

The $328 \mathrm{keV}$ peak is a background peak, and its source has been identified as ${ }^{207} \mathrm{Bi}$. In future experiments, this peak will be eliminated by improved shielding of the germanium detector. The peaks at 111.0, 121.0, and $148.4 \mathrm{keV}$ are as yet unidentified, but since this study requires signals from ${ }^{180} \mathrm{Ta}$ and ${ }^{178 m}$ Ta alone, they do not cause any ambiguity.

As well as measuring the energies of the $\gamma$ rays emitted from the tantalum sample, another method for isotope identification is to measure the decay of the sample. Since radioisotopes all have characteristic half lives, a positive measurement coupled with the energy measurements increases the reliability of the identification.

To measure the decay of the $\gamma$-ray activity of the sample, four measurements lasting $1 \mathrm{~h}$ were taken. The areas of the peaks were then measured as a function of time. The most useful peaks are at $103 \mathrm{keV}\left({ }^{180} \mathrm{Ta}\right)$ and $426 \mathrm{keV}\left({ }^{178 m} \mathrm{Ta}\right)$ since these are unique to the two isotopes in question, and occur in areas of the spectrum which are uncontaminated from background contributions. Figure 4 shows the areas of these peaks as a function of time, where $t=0$ is the time of laser irradiation. From this decay data, the half lives of the peaks were established. The $103 \mathrm{keV}$ peak half life was found to be $7.4 \pm 0.3 \mathrm{~h}$. The accepted value for ${ }^{180} \mathrm{Ta}$ is $8.1 \mathrm{~h}$. The decay was only measured over one half life-to yield more accurate results the decay should have been monitored for longer, but this was not possible due to experimental constraints. A more detailed measurement will be carried out in future studies. The half-life measurement obtained however, coupled with the energy measurement of the peak, is more than adequate to identify the source of this radiation as ${ }^{180} \mathrm{Ta}$, produced via the reaction ${ }^{181} \mathrm{Ta}(\gamma, n){ }^{180} \mathrm{Ta}$. The half life of the $426 \mathrm{keV}$ peak was found to be $2.2 \pm 0.2 \mathrm{~h}$ (accepted value for ${ }^{178 m} \mathrm{Ta}=2.36 \mathrm{~h}$ ). This provides unambigu- 


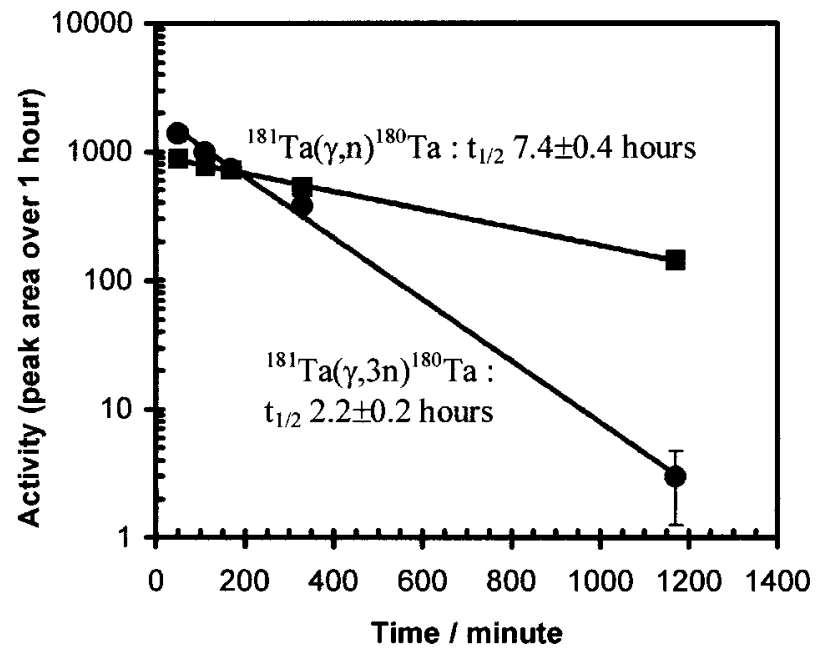

FIG. 4. Decay plots of the two peaks in question. Half-life measurements confirm the presence of ${ }^{180} \mathrm{Ta}$ and ${ }^{178 m} \mathrm{Ta}$.

ous evidence that the source of this peak is ${ }^{178 m} \mathrm{Ta}$, produced via the reaction ${ }^{181} \mathrm{Ta}(\gamma, 3 n){ }^{178 m} \mathrm{Ta}$. The result obtained is closer to the accepted value than for the ${ }^{180} \mathrm{Ta}$ peak because the decay was monitored for almost 10 half lives, as opposed to just one for the previous measurement.

The long analysis time described is only to ascertain the procedure, whereas once established, temperature measurements can be made in a few minutes and certainly before the next pulse in large laser systems.

Now that the presence of ${ }^{180} \mathrm{Ta}$ and ${ }^{178 m} \mathrm{Ta}$ was established, the activity ratio of these isotopes was measured. This was done by measuring the areas of the peaks in question and taking into account their relative intensities which are $0.78 \%$ for the $103 \mathrm{keV}$ peak and $97 \%$ for the $427 \mathrm{keV}$ peak. $^{27,28}$ In addition, the efficiency of the detector had to be included in the calculation, since this varied with incident energy.

Another important factor to take into consideration is the absorption of $\gamma$ rays in the tantalum target. The mass attenuation coefficient varies with $\gamma$-ray energy,${ }^{30}$ and the fraction of $\gamma$ rays absorbed was calculated for the relevant energies. The importance of making this correction was made obvious by looking at the 426 and $213.4 \mathrm{keV}$ peaks. According to the decay scheme of ${ }^{178 m} \mathrm{Ta}$, these should be almost equal in intensity. After taking into account the detector efficiency, it was found that the area of the $426 \mathrm{keV}$ peak was greater than that of the $213.4 \mathrm{keV}$ peak by a factor of 1.7 . After correcting for absorption effects, the peak areas were found to differ by a factor of 1.1. Correcting for the 426 and $103 \mathrm{keV}$ peaks in addition to branching ratios and the detector efficiency gave an experimentally measured ${ }^{178 m} \mathrm{Ta} /{ }^{180} \mathrm{Ta}$ activity ratio of $(1.78 \pm 0.44) \times 10^{-3}$. This measurement was then fitted to the theoretical activity ratio calculated as a function of $k T$.

The theoretical ${ }^{178 m} \mathrm{Ta} /{ }^{180} \mathrm{Ta}$ activity ratio was calculated in the following way: the relativistic Maxwellian electron energy distribution in $\eta$ dimensions is given by

$$
N(E) \propto \gamma\left(\gamma^{2}-1\right)^{(\eta / 2)-1} e^{-(E / k T)},
$$

where $N(E)$ is the number of electrons of energy $E, \gamma=1$ $+E / m c^{2}, k$ is Boltzmann's constant, and $T$ is the electron

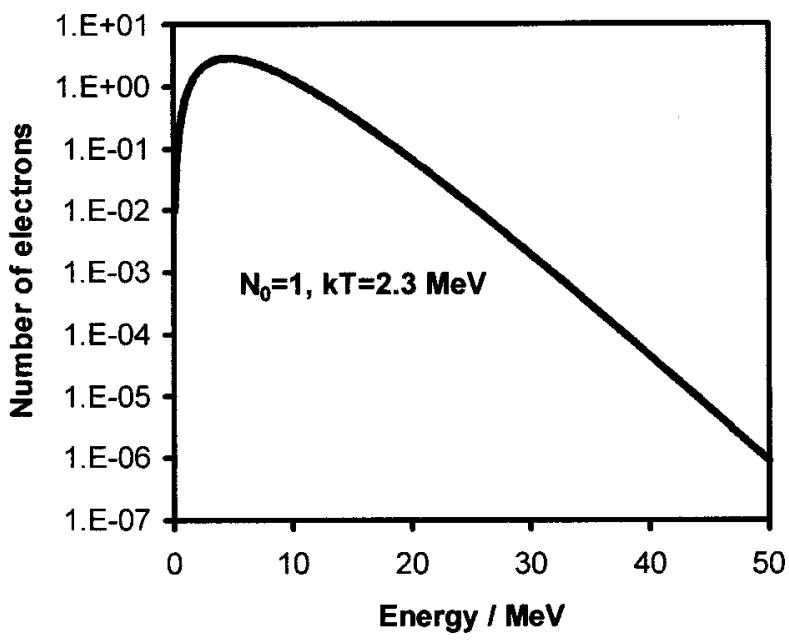

FIG. 5. The shape of the relativistic electron energy distribution for $N_{0}$ $=1, k T=2.3 \mathrm{MeV}$.

temperature. In the ultrarelativistic limit and threedimensional space, this tends to the form ${ }^{1,4,26}$

$$
N(E)=N_{0} E^{2} e^{-(E / k T)},
$$

where $N_{0}$ is a constant. Figure 5 shows this distribution for $E=0-50 \mathrm{MeV}, k T=2.3 \mathrm{MeV}$, and $N_{0}=1$.

Electrons of energy $E \mathrm{MeV}$ produce a $\gamma$-ray spectrum in tantalum via bremsstrahlung. This spectrum was calculated using an analytical representation for bremsstrahlung spectra developed by Findlay. ${ }^{31}$ Figure 6 shows the calculated bremsstrahlung yield for photons produced along the laser direction by electrons of energy 10 and $25 \mathrm{MeV}$.

The induced activity of a sample when bombarded with high-energy photons may be obtained from

$$
A=\left(\frac{\ln (2)}{t_{1 / 2}}\right) \sum_{E_{\gamma}} N\left(E_{\gamma}\right) \sigma_{\gamma}\left(E_{\gamma}\right) N
$$

where $t_{1 / 2}$ is the half life of the radioisotope, $N_{\gamma}\left(E_{\gamma}\right)$ is the number of photons in the energy bin of width $0.5 \mathrm{MeV}$ at energy $E_{\gamma}, \sigma_{\gamma}\left(E_{\gamma}\right)$ is the photonuclear reaction cross section at energy $E_{\gamma}$, and $N$ is the number of nuclei present/cc. The results are shown in Fig. 7. The experimentally mea-

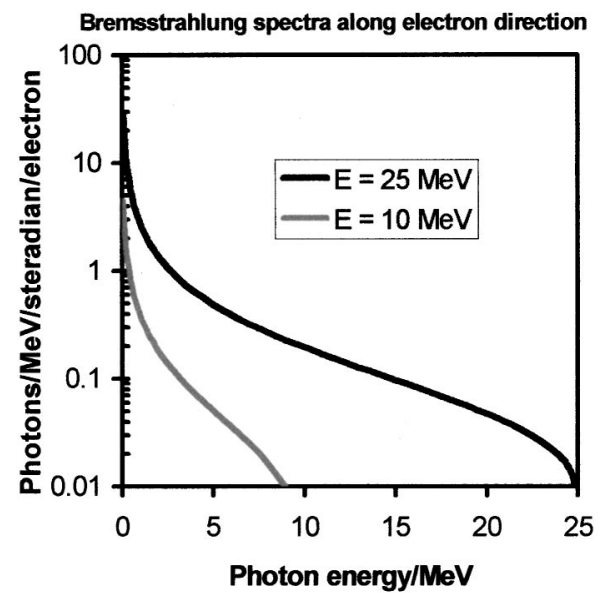

FIG. 6. Bremsstrahlung spectra along relativistic electron beam direction of electrons of energy 10 and $25 \mathrm{MeV}$. 


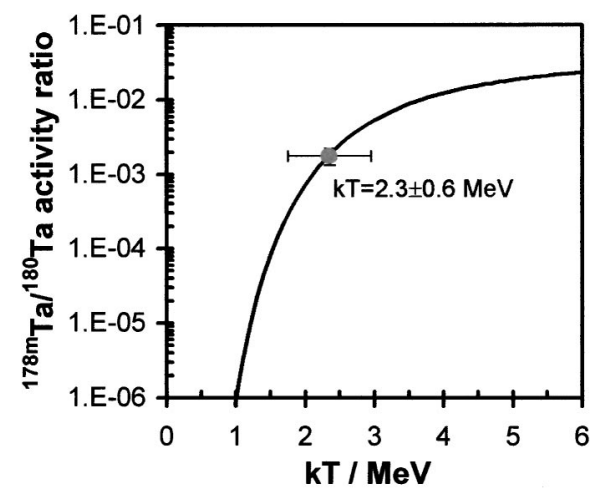

FIG. 7. Theoretical ${ }^{178 m} \mathrm{Ta} /{ }^{180} \mathrm{Ta}$ activity ratio as a function of $k T$. The experimentally measured ratio corresponds to the $k T$ value of $2.3 \pm 0.6 \mathrm{MeV}$.

sured activity ratio of $(1.78 \pm 0.44) \times 10^{-3}$ corresponds to $k T=2.3 \pm 0.6 \mathrm{MeV}$ on this plot. The intensity on target was $\sim 10^{20} \mathrm{~W} \mathrm{~cm}^{-2}$. This measurement is in agreement with the measurement made using the $\mathrm{Cu} / \mathrm{C}$ technique ${ }^{4}$ at a slightly lower intensity. This is in reasonable agreement with the electron spectrum reported by Cowan et al. ${ }^{5}$-a rough analysis of this spectrum shows that the temperature is $\sim 3.5 \mathrm{MeV}$ for an intensity greater than $10^{20} \mathrm{~W} \mathrm{~cm}^{-2}$. Measurements of proton beam temperatures ${ }^{32}$ give $k T=5-6 \mathrm{MeV}$. The measurement is in good agreement with the $\mathbf{j} \times \mathbf{B}$ ponderomotive model described by Wilks et al. ${ }^{33}$ and confirmed experimentally by Malka and Miquel, ${ }^{34}$ where, for $p$ polarization and oblique incidence, $k T$ is of the order $0.511\left(\sqrt{ }\left\{1+I \lambda^{2} / 1.37\right.\right.$ $\left.\left.\times 10^{18} / 2\right\}-1\right) \mathrm{MeV}$. For $I \lambda^{2}=10^{20} \mathrm{~W} \mathrm{~cm}^{-2} \mu \mathrm{m}^{2}, k T$ is calculated to be $2.6 \mathrm{MeV}$. Measurements made by Santala et $a .^{22}$ using a similar technique at a preliminary level gave a $k T$ value of $4.4 \mathrm{MeV}$ at similar intensities, although this experiment involved a laser-gas interaction which is known to give higher electron temperatures than laser-solid interactions. ${ }^{35}$ If the data were not corrected for absorption effects, the $k T$ value measured would be of the order of 5 $\mathrm{MeV}$ which, for a laser-solid interaction at intensities up to $10^{20} \mathrm{~W} \mathrm{~cm}^{-2}$, would be higher than expected and observed in other measurements. Therefore, the measurement made appears to be reasonable.

\section{DISCUSSION}

It has been shown that higher-order photonuclear reactions can be used to measure the electron temperature when an ultraintense laser pulse interacts with a solid tantalum target. Analysis of the specific peaks described is recommended since they are unambiguous for the isotopes ${ }^{180} \mathrm{Ta}$ and ${ }^{178 m}$ Ta. For higher laser intensities and hence higher electron and photon energies, this technique should still be applicable since one simply looks for evidence of higher order reactions using the same spectroscopic technique and choosing thoughtfully the reactions that will yield unambiguous peaks. In particular, ${ }^{181} \mathrm{Ta}(\gamma, 4 n){ }^{177} \mathrm{Ta}(Q=29.0 \mathrm{MeV})$ and ${ }^{181} \mathrm{Ta}(\gamma, 5 n){ }^{176} \mathrm{Ta}(Q=37.3 \mathrm{MeV})$ look like very useful reactions for this technique. ${ }^{177} \mathrm{Ta}$ has a half life of $2.36 \mathrm{~d}$, and unambiguous $\gamma$-ray lines at 113.0 and $208.4 \mathrm{keV}$. ${ }^{176} \mathrm{Ta}$ has a half life of $8.08 \mathrm{~h}$, and a cascade of transitions with the highest energy $1115.9 \mathrm{keV}$, plus many others of lower energy with the same intensity, yielding a fingerprint similar in nature to that of ${ }^{178 m} \mathrm{Ta}$ shown above, but with different $\gamma$-ray lines. This technique can provide temperature measurements on a shot-to-shot basis, even while other experiments (e.g., angular distribution measurements) are being conducted. The technique is also applicable for gas target interactions if the tantalum sample is placed in the path of the fast electron beam. Other activation samples may also prove useful, and a full study involving higher order reactions in other high $Z$ materials is planned for the future in order to find the most suitable material for the electron spectra obtained at various laser intensities.

\section{ACKNOWLEDGMENTS}

The authors would like to acknowledge the support of the engineering and target area staff of the Central Laser Facility of the Rutherford Appleton Laboratory and useful discussions with Marko Santala. This work was supported by the U.K. Engineering and Physical Sciences Research Council.

${ }^{1}$ P. A. Norreys et al., Phys. Plasmas 6, 2150 (1999).

${ }^{2}$ T. W. Phillips, M. D. Cable, T. E. Cowan, S. P. Hatchett, E. A. Henry, M. H. Key, T. Perry, C. Sangster, and M. A. Stoyer, Rev. Sci. Instrum. 70, 1213 (1999).

${ }^{3}$ K. W. D. Ledingham and P. A. Norreys, Contemp. Phys. 40, 367 (1999).

${ }^{4}$ K. W. D. Ledingham et al., Phys. Rev. Lett. 84, 899 (2000).

${ }^{5}$ T. E. Cowan et al., Phys. Rev. Lett. 84, 903 (2000).

${ }^{6}$ K. Boyer, T. S. Luk, and C. K. Rhodes, Phys. Rev. Lett. 60, 557 (1988).

${ }^{7}$ S. J. Gitomer, R. D. Jones, F. Begay, A. W. Ehler, J. F. Kephart, and R. Kristal, Phys. Fluids 29, 2679 (1986)

${ }^{8}$ Y. Kishimoto, K. Mima, T. Watanabe, and K. Nishikawa, Phys. Fluids 26, 2308 (1983).

${ }^{9}$ F. N. Beg et al., Phys. Plasmas 4, 447 (1997).

${ }^{10}$ A. P. Fews, P. A. Norreys, F. N. Beg, A. R. Bell, A. E. Dangor, C. Danson, P. Lee, and S. J. Rose, Phys. Rev. Lett. 73, 1801 (1994).

${ }^{11}$ E. L. Clark et al., Phys. Rev. Lett. 84, 670 (2000).

${ }^{12}$ E. L. Clark et al., Phys. Rev. Lett. 85, 1654 (2000).

${ }^{13}$ K. Krushelnick, E. L. Clark, Z. Najmudin, M. Salvati, M. I. K. Santala, M. Tatarakis, and A. E. Dangor, Phys. Rev. Lett. 83, 737 (1999).

${ }^{14}$ M. I. K. Santala et al., Appl. Phys. Lett. 78, 19 (2001).

${ }^{15}$ R. A. Snavely et al., Phys. Rev. Lett. 85, 2945 (2000).

${ }^{16}$ I. Spencer et al., Nucl. Instrum. Methods Phys. Res. B 183, 449 (2001).

${ }^{17}$ A. Maksimchuk, S. Gu, K. Flippo, and D. Umstadter, Phys. Rev. Lett. 84, 4108 (2000).

${ }^{18}$ K. Nemoto, A. Maksimchuk, S. Banerjee, K. Flippo, D. Umstadter, and V. Y. Bychenkov, Appl. Phys. Lett. 78, 595 (2001).

${ }^{19}$ M. Zepf et al., Phys. Plasmas 8, 2323 (2001).

${ }^{20}$ M. I. K. Santala et al., Phys. Rev. Lett. 84, 1459 (2000).

${ }^{21}$ T. E. Cowan et al., Nucl. Instrum. Methods Phys. Res. A 455, 130 (2000).

${ }^{22}$ M. I. K. Santala et al., Phys. Rev. Lett. 86, 1227 (2001).

${ }^{23}$ C. Danson et al., J. Mod. Opt. 45, 1653 (1998).

${ }^{24}$ M. D. Perry and G. Mourou, Science 264, 917 (1994).

${ }^{25}$ D. Strickland and G. Mourou, Opt. Commun. 56, 219 (1985).

${ }^{26}$ J. R. Davies, Phys. Rev. E 65, 026407 (2002).

${ }^{27}$ R. B. Firestone, V. S. Shirley, and C. M. Chu, Table of Isotopes, 8th ed. (1998).

${ }^{28}$ J. Magill, NUCLIDES 2000, computer program.

${ }^{29}$ EXFOR www nuclear reaction database, http://iaeand.iaea.or.at/exfor/

${ }^{30}$ NIST x-ray mass attenuation coefficients, http://aeldata.phy.nist.gov/ PhysRefData/XrayMassCoef/cover.html

${ }^{31}$ D. J. S. Findlay, Nucl. Instrum. Methods Phys. Res. A 276, 598 (1989).

${ }^{32}$ M. Roth et al., Phys. Rev. Lett. 86, 436 (2001).

${ }^{33}$ S. C. Wilks, W. L. Kruer, M. Tabak, and A. B. Langdon, Phys. Rev. Lett. 69, 1383 (1992).

${ }^{34}$ G. Malka and J. L. Miquel, Phys. Rev. Lett. 77, 75 (1996).

${ }^{35}$ D. Umstadter, Phys. Plasmas 8, 1774 (2001). 